\title{
Homoepitaxial Growth of Pt on Pt(100)-hex: Effects of Strongly Anisotropic Diffusion and Finite Island Sizes
}

\author{
Linderoth, T.R.; Mortensen, Jens Jørgen; Jacobsen, Karsten Wedel; Lægsgaard, E.; Stensgaard, I.; \\ Besenbacher, Flemming
}

Published in:

Physical Review Letters

Link to article, DOI:

10.1103/PhysRevLett.77.87

Publication date:

1996

Document Version

Publisher's PDF, also known as Version of record

Link back to DTU Orbit

Citation (APA):

Linderoth, T. R., Mortensen, J. J., Jacobsen, K. W., Lægsgaard, E., Stensgaard, I., \& Besenbacher, F. (1996). Homoepitaxial Growth of Pt on Pt(100)-hex: Effects of Strongly Anisotropic Diffusion and Finite Island Sizes.

Physical Review Letters, 77(1), 87-90. https://doi.org/10.1103/PhysRevLett.77.87

\section{General rights}

Copyright and moral rights for the publications made accessible in the public portal are retained by the authors and/or other copyright owners and it is a condition of accessing publications that users recognise and abide by the legal requirements associated with these rights.

- Users may download and print one copy of any publication from the public portal for the purpose of private study or research.

- You may not further distribute the material or use it for any profit-making activity or commercial gain

- You may freely distribute the URL identifying the publication in the public portal 


\title{
Homoepitaxial Growth of Pt on Pt(100)-hex: Effects of Strongly Anisotropic Diffusion and Finite Island Sizes
}

\author{
T. R. Linderoth, ${ }^{1}$ J. J. Mortensen, ${ }^{2}$ K. W. Jacobsen, ${ }^{2}$ E. Lægsgaard, ${ }^{1}$ I. Stensgaard, ${ }^{1}$ and F. Besenbacher ${ }^{1}$ \\ ${ }^{1}$ CAMP, Institute of Physics and Astronomy, University of Aarhus, DK 8000 Aarhus C, Denmark \\ ${ }^{2}$ CAMP, Department of Physics, Technical University of Denmark, DK 2800 Lyngby, Denmark
}

(Received 22 January 1996)

\begin{abstract}
Nucleation and growth of $\mathrm{Pt}$ on the reconstructed $\mathrm{Pt}(100)$-hex surface was studied by scanning tunneling microscopy. A detailed autocorrelation analysis of island positions reveals direct evidence for strongly anisotropic diffusion, and from the island size distribution, which obeys a simple scaling relation, we conclude that the mobility of dimers is negligible. Finally, kinetic Monte Carlo simulations, incorporating anisotropy in diffusion and finite island sizes, yield new insight into how these two factors affect the island size distribution and the scaling behavior of island density with temperature and deposition rate. [S0031-9007(96)00572-8]

PACS numbers: 61.16.Ch, 68.35.Fx, 68.55.-a
\end{abstract}

The understanding of the initial stages of metal on metal growth has improved considerably in the last few years not least because of detailed scanning tunneling microscopy (STM) studies with atomic or near-atomic resolution [1-3]. Especially the island nucleation and growth scenario for simple isotropic systems [4] has been put on a much firmer basis, while the understanding of anisotropic and reconstructed systems [5-8] is less well founded.

In this Letter, we present comprehensive STM measurements on the submonolayer homoepitaxial nucleation and growth of Pt on the reconstructed Pt(100)-hex surface, which exhibits a highly anisotropic surface morphology. Island positions, sizes, and number densities have been determined over a wide range of deposition rate $(R)$ and temperature $(T)$, and these data are compared with the results of kinetic Monte Carlo (kMC) simulations. It is shown how a thorough statistical analysis of the experimental data, in the form of autocorrelation functions for island positions and normalized island size distributions, in a unique way supplements the information usually extracted from the scaling of island density with $R$ and $T$. New insight into the way in which highly anisotropic diffusion in combination with finite island sizes influences the scaling of island density and the island size distribution is presented. More specifically, the autocorrelation analysis reveals that the islands are anisotropically distributed, implying a highly anisotropic surface diffusion of $\mathrm{Pt}$ adatoms. The island size distributions, which by proper scaling collapse onto a single scaling curve, confirm that the diffusion is anisotropic, reveal an early onset of coalescence, and indicate a negligible dimer mobility. The island densities conform with the usual type of scaling law, $N_{x} \sim(R / h)^{\chi}$, where $h$ denotes an effective adatom hopping rate and $\chi$ is found to be 0.27 . From this result, it is concluded that the critical island size $i$ is 1 , corresponding to dimers being stable, and that the surface diffusion is described by an effective barrier $E_{d}=0.43 \mathrm{eV}$.
The experiments were performed in an ultrahighvacuum system equipped with an STM. The sample was sputter cleaned followed by annealing to $970 \mathrm{~K}$, resulting in terraces of several thousand $\AA$ width. Evaporation was performed from a resistively heated Pt filament, and the sample temperature was measured by a thermocouple mounted to the back of the sample. The evaporation rate was determined from the evaporation time, controlled by a shutter, and the deposited coverage, as determined from the STM images. Depositions were performed at five temperatures between 318 and $497 \mathrm{~K}$. At each temperature, several rates were used, corresponding to deposition times ranging from 10 to $1800 \mathrm{sec}$. For each set of deposition parameters $\sim 2000$ individual islands on large step-free terraces were analyzed.

$\mathrm{Pt}$ belongs to the class of late $5 d$ metals, the clean (100) surfaces of which reconstruct by forming a contracted quasihexagonal (hex) layer resting on top of the quadratic substrate [9-11]. Figure 1(a) shows an atomically resolved STM image of the $\mathrm{Pt}(100)$-hex surface. The hex layer is oriented such that there is alignment between the close-packed directions in the two layers (indicated by white line segment). The misfit between the two layers leads to a characteristic height modulation. (a)

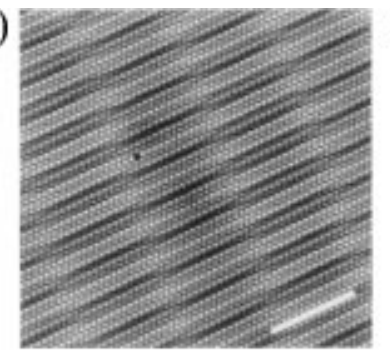

(b)

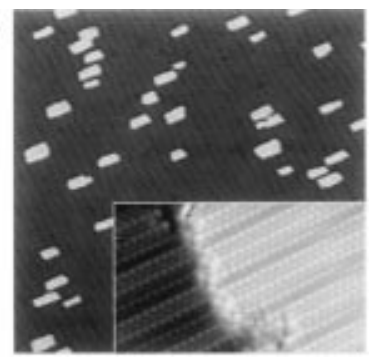

FIG. 1. STM images of (a) the Pt(100)-hex surface $(140 \times$ $140 \AA^{2}$ ), (b) monoatomically high islands obtained after Pt deposition $\left(R=2.3 \times 10^{-4} \mathrm{site}^{-1} \mathrm{sec}^{-1}, T=389 \mathrm{~K}, 1400 \times\right.$ $1400 \AA^{2}$ ). Inset: Edge of island, atomically resolved. 
Perpendicularly to the line, this height modulation has a periodicity of six top-layer atoms, and is seen as rowlike structures running parallel to the line, henceforth referred to as the direction of the reconstruction rows. Although the atoms in the topmost layer have a nearly hexagonal arrangement, which might indicate isotropic diffusion of adatoms, the highly anisotropic large-scale surface morphology of the $\mathrm{Pt}(100)$-hex phase points towards strongly anisotropic effects in the Pt adatom diffusion. Homoepitaxial growth on this surface is also interesting since the reconstruction is known to be lifted by the adsorption of various molecules $[9,10]$.

As shown in Fig. 1(b), deposition of a submonolayer amount of Pt on the Pt(100)-hex surface results in the formation of monoatomically high islands, which also exhibit the characteristic height modulation of the reconstruction. Initially $N_{x}$ increases with the Pt coverage until a saturation is reached. The subsequent measurements discussed here are made in the saturation regime at a coverage of $\approx 0.07 \mathrm{ML}$. The islands are compact, and at the higher temperatures they are of a regular rectangular shape with an aspect ratio (length/width) between 1 and 3, and lie oriented with their long edge parallel to the direction of the reconstruction rows. Since the Pt islands are found to be reconstructed, it is believed that a transformation from a quasihexagonal to a quadratic arrangement of the atoms takes place underneath a growing island.

The spatial correlations between the islands can be investigated quantitatively by considering the autocorrelation function for the island positions. This is constructed from all images corresponding to a given set of $(R, T)$ by superposing from a common origin all interisland vectors connecting the center of gravity for two islands. The autocorrelation function, as shown in Fig. 2(a), reveals that the density of interisland vectors is significantly reduced in the direction parallel to the reconstruction rows, thus demonstrating a clear anisotropy in the correlation of the island positions. This is exactly the expected behavior if adatom diffusion is highly anisotropic with the fast direction along the reconstruction rows. The central region in
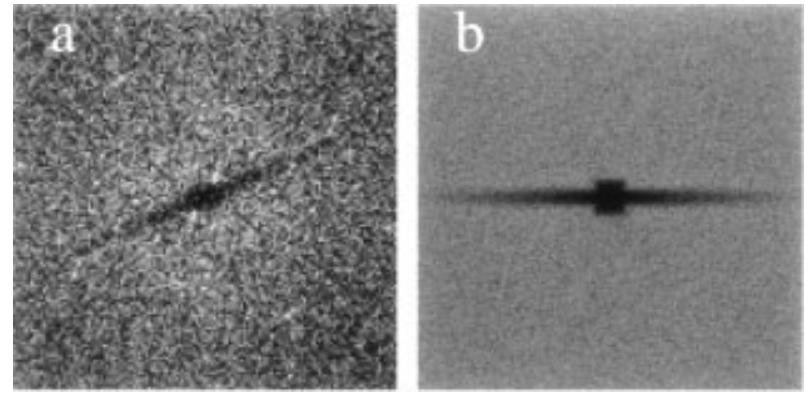

FIG. 2. Autocorrelation functions for island positions. (a) Experiment: $R=2.3 \times 10^{-4} \mathrm{site}^{-1} \mathrm{sec}^{-1}, T=389 \mathrm{~K}, 1600 \times$ $1600 \AA^{2}$. The regions with a reduced density of interisland vectors point exactly in the direction of the reconstruction rows. (b) Simulation: $h_{x} / h_{y}=1000, h_{x} / R=10^{9}, 261 \times 261$ sites. the histogram with no interisland vectors is mainly due to the finite sizes and shapes of the islands.

In Fig. 3(a), the island size distributions for different temperatures at a fixed rate are shown. If the island sizes are measured relative to the average island size $s_{\mathrm{av}}$, the normalized distributions possess a universal scaling behavior and collapse onto a single curve [8] $f\left(s / s_{\mathrm{av}}\right)$, as shown in Fig. 3(b). Such scaling has previously been discussed theoretically in both the isotropic $[8,12]$ and anisotropic cases [8] and confirmed experimentally [3] in the case of isotropic diffusion.

In order to gain insight into the effects of anisotropic diffusion and finite island sizes on the described observations, $\mathrm{kMC}$ simulations have been performed. $\mathrm{Pt}(100)$ hex has a very complex surface morphology, and most

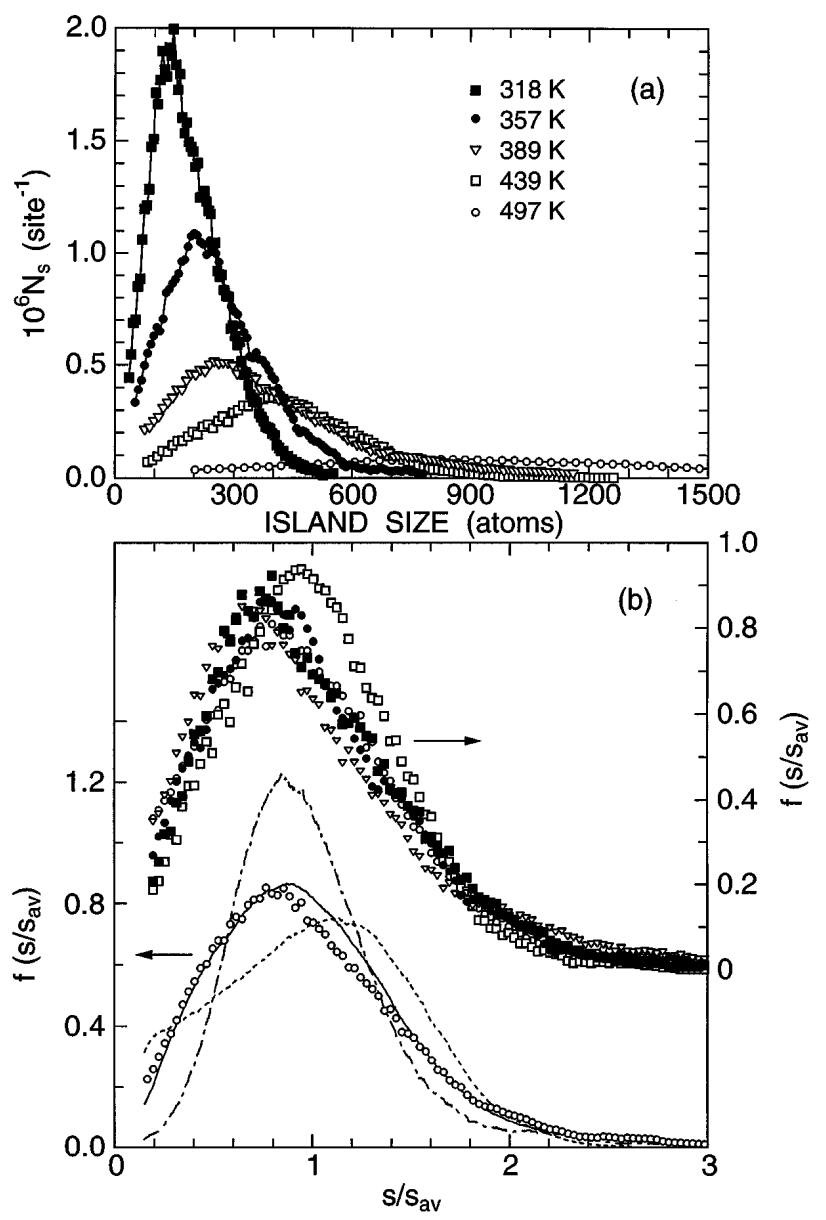

FIG. 3. (a) Island size distributions showing the density $N_{s}$ of islands of size $s\left(R=8 \times 10^{-3} \mathrm{site}^{-1} \mathrm{sec}^{-1}\right.$, temperatures as stated in figure). (b) Top: The same distributions as in (a), scaled according to the proposal of Ref. [8]. The fact that the $439 \mathrm{~K}$ result differs slightly might be ascribed to small fluctuations in the deposited coverage. Bottom: Comparison to simulations. Circles: Average over the five scaled distributions. Solid line: Strongly anisotropic diffusion $\left(h_{x} / h_{y}=1000\right.$, $\left.h_{x} / R=10^{9}\right)$. Dashed line: Isotropic diffusion $\left(h_{x} / h_{y}=1\right.$, $\left.h / R=10^{9}\right)$. Dash-dotted line: Strongly anisotropic diffusion of both monomers and dimers $\left(h_{x} / h_{y}=1000, h_{x} / R=10^{9}\right)$. 
likely the corrugation of the $\operatorname{Pt}(100)$ substrate gives rise to a distribution of energy barriers on the hex overlayer. We make no attempt at directly reproducing this. Instead we adopt the simplest model containing anisotropy in diffusion and compact islands of finite sizes. Adatoms are deposited on a square lattice at a rate $R$ and are allowed to jump to the four nearest neighbors with rates $h_{x}$, $h_{y}$ for jumps along the $x$ and $y$ directions, respectively, the anisotropy being controlled by the ratio $h_{x} / h_{y}$. Two adatoms will aggregate irreversibly upon encounter and thereby nucleate an island. Adatoms captured by an island are placed at an edge site of the island in such a way that islands are always compact and approximately square. The simulation is stopped at a coverage of $0.07 \mathrm{ML}$.

The experimentally determined autocorrelation function may be compared with one obtained from such simple kMC simulations. The autocorrelation plot in Fig. 2(b) was obtained from simulations with $h_{x} / R=10^{9}$ [13], and for an anisotropy of $h_{x} / h_{y}=1000$, which is the order of magnitude giving the best agreement with the experimentally obtained autocorrelation function.

The scaled island size distribution is also reproduced by the kMC simulations with $h_{x} / h_{y}=1000$ as shown in Fig. 3(b). The scaled distribution differs from the ones obtained earlier for isotropic diffusion [12] and, in a point-island model, for anisotropic diffusion [8]. Especially, the island size where the distribution takes on its maximum value is less than the average island size. This is mainly a result of coalescence due to the finite island sizes. For high diffusional anisotropies, the extent of an island in the direction of slow diffusion can already at rather small coverages become comparable to the diffusion length in that direction, giving rise to an earlier onset of coalescence as compared to the case of isotropic diffusion. This is indeed the case for $\operatorname{Pt}(100)$ hex, where the islands are compact with a relatively small aspect ratio. In the simulations, this effect can clearly be seen: By neglecting coalescence when evaluating the island sizes, the peak position in the scaled distribution moves very close to the average island size.

The island size distribution can also be used to establish whether the main diffusing objects are monomers or dimers (or even larger islands) [14]. Figure 3(b) shows the simulated island size distribution if dimers are assigned the same mobility as monomers. The resulting scaled island size distribution clearly has a much higher peak value than the observed distribution, and consequently we conclude that the mobility of dimers is negligible as compared to that of the monomers.

A change in the critical island size away from $i=1$ also affects the island size distribution. Simulated island size distributions for $i=0$ are seen to be monotonically decaying functions [12] as opposed to the presently observed distribution. This shows that nucleation is not due to pinning at impurities or special sites on the surface. The fact that $i \neq 0$ also rules out the possibility that nucleation is due to local "deconstruction" events, which is interesting keeping in mind that the reconstruction is lifted under a growing island. The changes for higher critical island sizes $(i=2,3, \ldots)$ are more delicate and to address these possibilities we turn to the scaling behavior of the saturation island density as a function of $(R, T)$.

From mean-field rate-equation descriptions of the nucleation process $[4,8]$, the saturation island density $N_{x}$ is expected to vary as

$$
N_{x} \sim\left(\frac{R}{\nu}\right)^{\chi} \exp (\chi E / k T), \quad E=E_{d}+E_{i} / i,
$$

where $E_{d}$ is the barrier for surface diffusion, $i$ and $E_{i}$ are the size and binding energy of the critical cluster, and $\nu$ is the prefactor for diffusion. By fitting the observed island densities to Eq. (1) we obtain $\chi=0.27 \pm 0.01$ and $E=$ $0.43 \pm 0.03 \mathrm{eV}$. As opposed to the usual plotting of $N_{x}$ versus $R$ for fixed $T$, we choose a more compact way of presenting the data shown in Fig. 4 . Here $N_{x}$ is plotted versus the parameter $R / \nu \exp (-E / k T)$, which we calculate using the fitted value for $E$ and $\nu=10^{13} \mathrm{sec}^{-1}$.

The scaling behavior of Eq. (1) has been analytically obtained in two cases: (i) for the well-studied case of isotropic diffusion the exponent is $\chi^{2 \mathrm{D}}=i /(i+2)[4,8]$, and (ii) for one-dimensional nucleation and growth, Evans and Bartelt [8] find $\chi^{1 \mathrm{D}}=i /(2 i+2)$. For the case of $i=1$, different scaling is thus to be expected with $\chi^{2 \mathrm{D}}=1 / 3$ and $\chi^{1 \mathrm{D}}=1 / 4$, respectively. Higher values of the critical island size lead in both cases to exponents considerably larger than the one seen experimentally and therefore we conclude that $i=1$ over the whole temperature interval examined (a change away from $i=1$ would prevent the observed collapse onto a single curve). Since $E_{1}=0$, the $E$ obtained from fitting by Eq. (1) can directly be associated with $E_{d}$. Because of the complicated surface morphology, the determined value must be interpreted as an effective or limiting barrier in the direction of fast diffusion.

Given that diffusion has been shown to be highly anisotropic, it would be tempting to jump to the

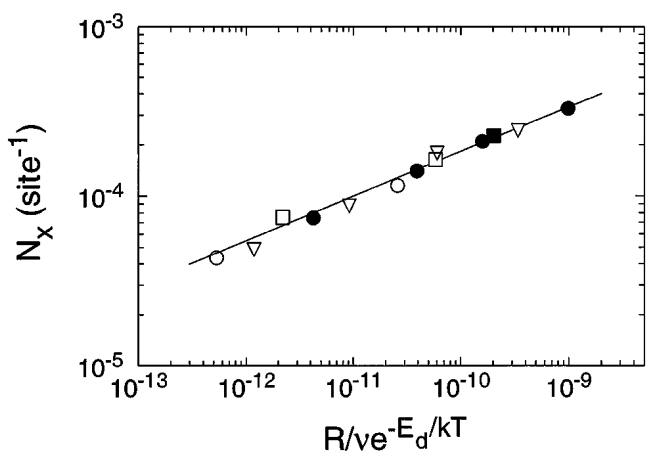

FIG. 4. Experimentally determined island densities, $N_{x}$, plotted against the parameter $R / \nu \exp (-E / k T)\left(E=E_{d}\right.$ since $i=1)$. The symbols correspond to the same deposition temperatures as in Fig. 3(a). 
conclusion that the measured value $\chi=0.27$ is a realization of the 1D nucleation and growth scenario. However, kMC simulations reveal that the exponent can be rather sensitive to several factors including both the degree of anisotropy and the island shapes and some further discussion is therefore warranted.

If we first neglect the island shapes and use a pointisland model in the simulations, the calculated exponent for infinitely anisotropic diffusion is seen to increase monotonically as a function of $h_{x} / R$ as also found in Ref. [8]. In the regime of very large $h_{x} / R$, it approaches the value $\chi=0.25$ consistent with the analytic rateequation results. However, including a small mobility perpendicularly to the direction of fast diffusion changes the exponent significantly; e.g., for an anisotropy of $h_{x} / h_{y}=1000$ it is increased to approximately $\chi=0.30$, as can be seen from an extrapolation to the experimentally relevant regime for $h_{x} / R\left(10^{9}-10^{12}\right)$.

The point-island model completely neglects the spatial extent of the islands. When the islands have a twodimensional shape, they will extend over, and may block, an increasing number of diffusion channels as they grow, reducing the nucleation probability by capturing an increasing number of adatoms. If this size-dependent capture probability is included in the rate-equation analysis, we obtain $\chi=1 / 3$ in the limit of infinitely anisotropic diffusion [15]. This analytic result is in agreement with simulations with square islands. From simulations with infinite anisotropy in the diffusion, we can extrapolate to find an exponent of $\chi=0.30 \pm 0.01$ in the experimentally relevant regime for $h_{x} / R$. If the anisotropy is $h_{x} / h_{y}=1000$, the value is increased to approximately $\chi=0.32$, and thus the value for the exponent gets very close to $1 / 3$ as for isotropic diffusion.

To summarize, the simulations reveal that in a pointisland model with anisotropic diffusion, values of $\chi$ between $1 / 4$ and $1 / 3$ are expected depending on the degree of anisotropy, whereas in a model with anisotropic diffusion and finite island sizes, a value for $\chi$ close to 0.3 is expected. The value $\chi=0.27$ obtained experimentally thus seems to be best reproduced in the point-island model, despite the fact that the islands are observed to be of finite extent. However, the blocking effect would be reduced if the diffusion on the $\operatorname{Pt}(100)$-hex surface is such that there is essentially one channel of fast diffusion for each 6-atom period of the reconstruction. We suggest that this is the case, and that the growth on this surface thus represents an intermediate case between the pointisland scenario and a situation with $2 \mathrm{D}$ islands.

It may be noted that Günther et al. [5] have studied homoepitaxial growth on $\mathrm{Au}(100)$-hex and found $\chi=0.37$ at variance with the result $\chi=0.27$ obtained here for $\mathrm{Pt}(100)$-hex. This difference could be due to significant dimer mobility in the Au case [16].

In conclusion, the submonolayer homoepitaxial growth of $\mathrm{Pt}$ on the reconstructed $\mathrm{Pt}(100)$-hex surface has been investigated with STM. The islands formed are compact, reconstructed, and two dimensional, and from the spatial distribution of islands highly anisotropic adatom diffusion is inferred. The effects of the combination of 2D islands and anisotropic diffusion have been investigated in simple $\mathrm{kMC}$ simulations. By comparing simulated island size distributions to the ones obtained experimentally, it is shown that coalescence occurs at an early stage in the growth process as well as it is demonstrated that dimer mobility is negligible. Finally, new theoretical results for the scaling of island densities with rate and temperature have been presented, and from the experimentally observed dependence, an effective barrier for surface diffusion is found and it is concluded that dimers are stable.

The Center for Atomic-scale Materials Physics (CAMP) is funded by the Danish National Research Foundation.

[1] H. Brune, H. Röder, C. Boragno, and K. Kern, Phys. Rev. Lett. 73, 1955 (1994).

[2] M. Bott, M. Hohage, M. Morgenstern, T. Micheley, and G. Comsa, Phys. Rev. Lett. 76, 1304 (1996).

[3] J. A. Stroscio and D. T. Pierce, Phys. Rev. B 49, 8522 (1994).

[4] J. A. Venables, Surf. Sci. 299/300, 798 (1994).

[5] S. Günther, E. Kopatzki, M. C. Bartelt, J. W. Evans, and R. J. Behm, Phys. Rev. Lett. 73, 553 (1994).

[6] J. P. Bucher, E. Hahn, P. Fernandez, C. Massobrio, and K. Kern, Europhys. Lett. 27, 473 (1994).

[7] Y. W. Mo, J. Kleiner, M. B. Webb, and M. G. Lagally, Phys. Rev. Lett. 66, 1998 (1991).

[8] J. W. Evans and M. C. Bartelt, J. Vac. Sci. Technol. A 12, 1800 (1994).

[9] A. Hopkinson, J. M. Bradley, X. Guo, and D. A. King, Phys. Rev. Lett. 71, 1597 (1993).

[10] A. Borg, A. M. Hilmen, and E. Bergenen, Surf. Sci. 306, 10 (1994).

[11] V. Fiorentini, M. Methfessel, and M. Scheffler, Phys. Rev. Lett. 71, 1051 (1993).

[12] J. G. Amar and F. Family, Phys. Rev. Lett. 74, 2066 (1995).

[13] For $h_{x} / R>10^{9}$ the kMC simulations become very time consuming. The simulated autocorrelation function and island size distributions have been obtained using the parameters $h_{x} / R=10^{9}$ and $h_{x} / h_{y}=1000$ yielding an island density of $N_{x}=6.4 \times 10^{-4}$, approximately one order of magnitude higher than the experimentally observed island densities. However, a scaling regime exists for $h_{x} / R>10^{8}$, where the autocorrelation function exhibits invariance with respect to $h_{x} / R$ if it is scaled with a length $N_{x}^{-1 / 2}$. Similarly, the island size distributions show perfect collapse in this regime.

[14] L. Bardotti, P. Jensen, A. Hoareau, M. Treilleux, and B. Cabaud, Phys. Rev. Lett. 74, 4694 (1995).

[15] J. J. Mortensen, M.S. thesis, 1995; J.J. Mortensen et al. (to be published).

[16] M. C. Bartelt, S. Günther, E. Kopatzki, R. J. Behm, and J. W. Evans, Phys. Rev. B 53, 4099 (1996). 